Redoxsignaling

\title{
NADPH-Oxidasen und HIF: zentrale Elemente der Redoxhomöostase
}

ANDREAS PETRY, AGNES GÖRLACH

EXPERIMENTELLE UND MOLEKULARE KINDERKARDIOLOGIE, DEUTSCHES HERZZENTRUM MÜNCHEN AN DER TU MÜNCHEN

\section{Reactive oxygen species (ROS) are not only toxic agents but also} potent signaling molecules. NADPH oxidases are the sole enzymes to generate ROS playing an important role in redox signaling. They closely interact in several ways with hypoxia-inducible transcription factors of the HIF family. This signaling network is active under normoxic and hypoxic conditions as an important regulator of redox homeostasis. Members of the NADPH oxidases-HIF axis appear as interesting therapeutic targets for various disorders characterized by an impaired redox homeostasis.

DOI: $10.1007 / \mathrm{s} 12268-021-1539-\mathrm{Z}$ (C) Die Autoren 2021
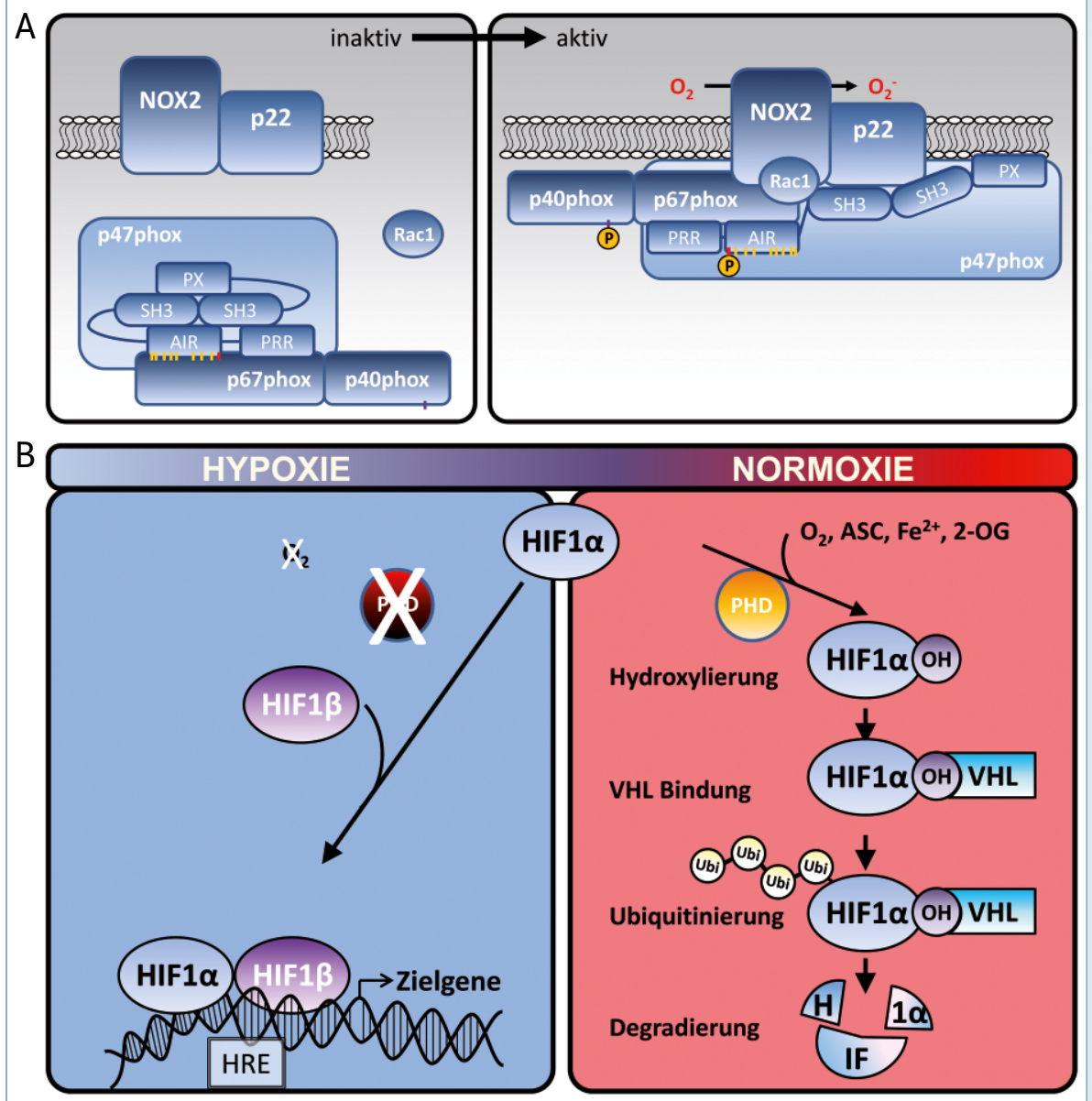

Reaktive Sauerstoffspezies (reactive oxygen species, ROS) spielen eine wichtige Rolle bei einer Vielzahl von biologischen Prozessen. Pathologisch erhöhte Werte führen zu oxidativem Stress bis hin zum Zelltod. ROS sind jedoch auch wichtige Signalmoleküle, die bei einer Vielzahl von zellulären Prozessen eine große Rolle spielen.

$\mathrm{Zu}$ den ROS gehören u. a. Sauerstoffradikale, wie das Superoxidanionradikal und das Hydroxylradikal, aber auch Nichtradikale wie Singulettsauerstoff und Wasserstoffperoxid [1]. ROS werden in den Mitochondrien als unspezifische Beiprodukte innerhalb der Atmungskette produziert. Auch andere Enzyme, wie Xanthinoxidase, Lipoxygenasen oder die NO-Synthase, können als Beiprodukte
4 Abb. 1: NADPH-Oxidase und HIF1. A, Im Ruhezustand sind die Komponenten der NADPHOxidase, das membranständige Flavocytochrom b558 mit seinen Untereinheiten NOX2 und p22phox, der cytosolische Komplex der regulatorischen Untereinheiten p47phox, p67phox und p40phox sowie die GTPase Rac 1 räumlich getrennt. Stimulation führt zur Phosphorylierung von S379 (rot) und anderen Serinen (gelb) in der autoinhibitorischen Region (AIR) von p47phox. Eine Konformationsänderung erlaubt die Bindung der p47phox SH3-Domänen an p22phox und zusammen mit dem aktiven Rac-GTP die Interaktion von p67phox mit NOX2. Diese Formation ermöglicht die Bildung von Superoxidanionradikalen. Rot: p47phox-S379; gelb: weitere phosphorylierbare Serine von p47phox (S303, S304. S315, S320, S328, S345, S348, S359, S370), violett: p40phox-T154. B, Unter Normoxie wird HIF $1 \alpha$ durch PHD in Abhängigkeit von der verfügbaren Sauerstoffkonzentration hydroxyliert. Die E3-Ubiquitinligase pVHL bindet an die hydroxylierten Proline. Dies führt zu Ubiquitinierung und proteosomaler Degradation. Sauerstoffmangel (Hypoxie) hemmt die Aktivität der PHD und den proteosomalen Abbau. HIF $1 \alpha$ wird stabilisiert, wandert in den Zellkern und bindet zusammen mit seinem Partner HIF $1 \beta$ an hypoxia response elements (HRE) von Zielgenen. ASC: Ascorbat; $\mathrm{Fe}^{2+}$ : Eisen(2)Ion; 2-OG: 2-Oxoglutarat; Ubi: Ubiquitin; VHL: von-HippelLindau-Protein; PHD: Prolylhydroxylase; AIR: autoinhibitorische Region; PRR: Prolin-rich region; SH3: Src-homology 3; PX: phox homology. 
oder unter pathologischen Bedingungen ROS bilden [1].

Dagegen gibt es mit der Familie der NADPH-Oxidasen auch spezialisierte Enzyme, deren einzige Funktion die Bildung von ROS ist. Die erste NADPH-Oxidase wurde in Phagozyten entdeckt, wo sie für den oxidativen oder respiratorischen Burst bei der Abwehr von Bakterien als Komponente der unspezifischen Immunabwehr verantwortlich ist. Dabei wird die NADPH-Oxidase durch Interaktion mit Pathogenen im Phagosom aktiviert und generiert große Mengen an Superoxid, das zur Tötung der Bakterien beiträgt. Fällt die Funktion der phagozytären NADPH-Oxidase durch Mutationen aus, kommt es zu einem schweren Immundefekt, der chronischen oder septischen Granulomatose. Patienten leiden an schweren, oft lebensgefährlichen Infektionen mit Bakterien und Pilzen [2].

\section{Aufbau und Regulierung der phagozytären NADPH-Oxidase}

Die phagozytäre NADPH-Oxidase besteht aus den membranständigen Proteinen NOX2 (früher auch gp91phox genannt) und p22phox, die zusammen das (Flavo-)Cytochrom b558 bilden. Das NOX-Protein beinhaltet den katalytischen Kern des Enzyms mit sechs transmembranären $\alpha$-Helices, die zwei Häme binden, und einer C-terminalen cytosolischen Dehydrogenase-Domäne, die Bindungsstellen für FAD (Flavinadenindinukleotid) und NADPH enthält [2].

Die Aktivierung der phagozytären NADPH-Oxidase ist sehr eng reguliert, um einer ungewollten Bildung von Superoxid und deren toxischen Konsequenzen vorzubeugen. Sie erfordert die Interaktion des Cytochroms b558 mit den regulatorischen Proteinen p40phox, p47phox und p67phox, die sich im inaktiven Zustand in einem Komplex im Cytosol befinden und, je nach Zelltyp, den kleinen G-Proteinen Rac1 oder Rac2 [2].

Zur Aktivierung muss zunächst die autoinhibitorische Region (AIR) von p47phox, die die beiden SH3-Domänen enthält, welche die Bindung an das Cytochrom b558 vermitteln, durch Serinphosphorylierung entriegelt werden. Von den insgesamt zehn verschiedenen Serinen, die phosphoryliert werden (S303, S304, S315, S320, S328, S345, S348, S459, S370, S379), ist insbesondere S379 wichtig, da dessen Deaktivierung im Gegensatz zu den anderen Phosphorylierungsstellen zu einem vollständi- gen Funktionsverlust der phagozytären NADPH-Oxidase führt. Durch die Phosphorylierung kommt es zu einer Konformitätsänderung von p47phox, die eine Bindung der SH3-Domänen an p22phox und damit eine Interaktion des cytosolischen Komplexes mit dem Cytochrom b558 ermöglicht. T154-Phosphorylierung von p40phox trägt ebenfalls zur Assemblierung und Aktivierung der NADPH-Oxidase bei [3].

Der eigentliche Aktivator der NADPHOxidase ist p67phox, dessen Interaktion mit aktiviertem Rac-GTP die Bindung an und eine Konformationsänderung von NOX2 fördert (Abb. 1A, [2]). Dies ermöglicht den transmembranären Elektronentransfer von NADPH über FAD und die Hämgruppen auf molekularen Sauerstoff und damit die Bildung von Superoxid.

\section{NADPH-Oxidasen in nicht- phagozytären Zellen}

Neben der „klassischen“ phagozytären NADPH-Oxidase mit NOX2 als katalytischer Untereinheit gibt es noch weitere NADPH-Oxidasen, die anstelle von NOX2 deren Homologe NOX1, NOX3, NOX4 und beim Menschen noch NOX5 als namensgebende katalytische Untereinheit besitzen [2]. Diese NADPH-Oxidasen unterscheiden sich auch durch ihre Aktivierungsmechanismen [4]. NOX1 und NOX3 sind - ähnlich wie NOX2 - auf cytosolische Untereinheiten, p47phox, p67phox bzw. deren Homologe NOXO1 und NOXA1, sowie auf Rac zur Aktivierung angewiesen. NOX4 dagegen benötigt lediglich p22phox und ist konstitutiv aktiv. Seine Regulation erfolgt vorwiegend auf Expressionsebene. NOX5 ist eine durch Calcium aktivierbare NADPH-Oxidase, die zwar - wie unser Labor zeigen konnte - mit p22phox interagieren kann, p22phox jedoch nicht zur Superoxidproduktion benötigt [5]. Daneben gibt es noch die beiden NOX-Homologe DUOX1/2 (für Dual-Oxidase1/2), die am N-Terminus eine extrazelluläre Peroxidasedomäne besitzen, wodurch sie $\mathrm{H}_{2} \mathrm{O}_{2}$ produzieren.

Mitglieder der NOX-Familie kommen in praktisch allen Gewebetypen vor, wobei das Expressionsmuster zelltypspezifisch variiert [2]. Während Phagozyten lediglich NOX2 exprimieren, findet man z. B. in Endothelzellen neben NOX2 in unterschiedlichen Mengen auch NOX1, NOX4, NOX5 und sogar DUOX [5, 6]. Warum eine Zelle unterschiedliche NOX-Enzyme besitzt, ist bisher noch nicht verstanden.

\section{Hier steht eine Anzeige.}

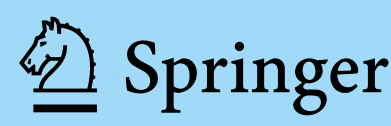




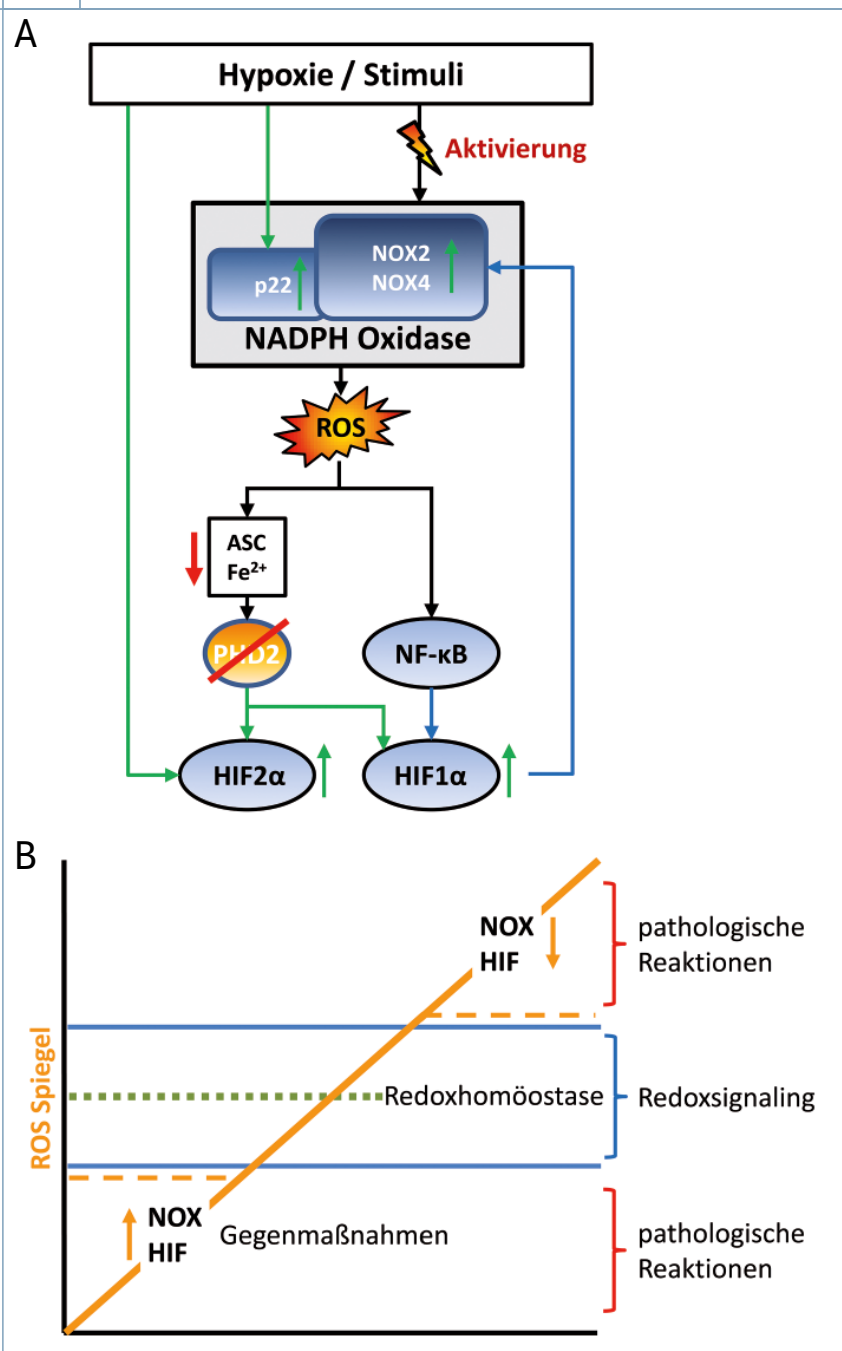

Im Gegensatz zu Phagozyten ist die ROSProduktion durch NADPH-Oxidasen in nichtphagozytierenden Zellen wesentlich geringer und wird durch zahlreiche Stimulatoren und pathophysiologische Zustände aktiviert und reguliert. In diesen Zellen übernehmen NADPH-Oxidasen und ROS eine wichtige Aufgabe als sekundäre Botenstoffe beim Redoxsignaling. Sie können u. a. durch die (reversible) Oxidation von Cystein-gebundenen Thiolgruppen spezifische Funktionsänderungen von Redoxproteinen wie Enzymen oder Transkriptionsfaktoren vermitteln und auch epigenetische Mechanismen regulieren $[1,7,8]$.

\section{Aufbau und Regulation des HIF- Systems}

NADPH-Oxidasen spielen auch eine interessante Rolle in der Regulation der Sauerstoffhomöostase durch Interaktion mit den Hypoxie-induzierbaren Transkriptionsfaktoren der HIF(hypoxia inducible factor)-Familie, den Schlüsselfaktoren der zellulären Antwort auf Hypoxie. Diese Heterodimere bestehen aus einer induzierbaren $\alpha$-Untereinheit (HIF $1 \alpha$, HIF $2 \alpha$, HIF $3 \alpha$ ) und einer konstitutiv exprimierten $\beta$-Untereinheit (HIF1 $\beta$, HIF2 $\beta$,

4 Abb. 2: Interaktion von NADPH-Oxidasen und HIF als Faktoren der Redoxhomöostase. A, Hypoxie sowie verschiedene Stimuli (z. B. Wachstumsund Gerinnungsfaktoren, Peptidhormone, Glukokortikoide) führen zur Induktion und/oder Aktivierung von NADPH-Oxidasen und einer erhöhten ROS-Bildung. Diese regulieren HIF $1 \alpha$ und HIF2 $\alpha$ entweder transkriptional über NFKBAktivierung oder über posttranslationale Stabilisierung durch Hemmung der PHD. NOX2 und NOX4 sind HIF1-Zielgene und p22phox wird unter Hypoxie stabilisiert. Grüne Pfeile: Induktion durch Stabilisierung; blaue Pfeile: transkriptionelle Induktion. B, Abweichungen von der Redoxhomöostase innerhalb eines ROS-Korridors führen zu Redoxsignaling und regulierten Anpassungsreaktionen. Stark verminderte oder erhöhte ROS-Spiegel führen zu pathologischen Reaktionen bis hin zum Zelltod. Modulation der NADPH-Oxidase-HIFAchse erlaubt ein Zurückführen der ROS-Spiegel in den ROS-Korridor zur Unterdrückung pathologischer Reaktionen. ASC: Ascorbat; $\mathrm{Fe}^{2+}$ : Eisen(2)Ion; NF-кB: nuclear factor 'kappa-light-chain-enhancer' of activated B-cells; ROS: reactive oxygen species.

auch als aryl hydrocarbon nuclear translocator, ARNT1/2 bekannt) [9].

Unter normoxischen Bedingungen wird die HIF $\alpha$-Untereinheit durch sauerstoffabhängige Prolylhydroxylasen (PHD1-3) an zwei spezifischen Prolinen hydroxyliert (bei humanem HIF1 $\alpha$ : P402 und P564). Die PHD benötigen als Ko-Faktoren Sauerstoff, Eisen (als $\left.\mathrm{Fe}^{2+}\right), \alpha$-Ketoglutarat (auch bekannt als 2-Oxaloglutarat, 2-OG) sowie Ascorbinsäure. Die hydroxylierten Proline werden vom von-Hippel-Lindau-Protein (pVHL) erkannt, das Bestandteil eines multimeren E3-Ubiquitinligase-Komplexes ist. Die anschließende Ubiquitinierung von HIF $\alpha$ führt dann zum proteasomalen Abbau. Eine Abnahme der Sauerstoffkonzentration beeinträchtigt die Aktivität der PHD, sodass die $\mathrm{HIF} \alpha$-Proteine unter Hypoxie stabilisiert werden und akkumulieren. Die HIF $\alpha$-Proteine wandern daraufhin in den Kern, wo sie mit der $\beta$-Untereinheit interagieren. Als Heterodimere binden sie an hypoxia response elements (HRE) in der genomischen DNA (Abb. 1B). Die mehr als 1.000 HIF-Zielgene umfassen u. a. Gene der Sauerstoffversorgung, des anaeroben Metabolismus und der Angiogenese [9].

\section{Regulation von HIF durch NADPH-Oxidasen}

NADPH-Oxidasen sind an der Regulation von HIF $\alpha$-Proteinen auf vielfältige Weise beteiligt (Abb. 2A). Bereits unter normoxischen Bedingungen induziert eine NADPH-Oxidaseabhängige Erhöhung der ROS Spiegel - beispielsweise durch Aktivierung des Enzyms durch Wachstumsfaktoren, Peptidhormone oder Gerinnungsfaktoren - die HIFo-Spiegel [10-12]. Dabei führen die erhöhten ROSSpiegel zu einer Verminderung der intrazellulären Menge an $\mathrm{Fe}^{2+}$ und Ascorbinsäure $[11,13]$, sodass diese Ko-Faktoren den PHD nicht mehr in ausreichender Weise zur Verfügung stehen. Die dadurch bedingte Abnahme der Aktivität der PHD führt aufgrund des verminderten proteasomalen Abbaus zu einer Akkumulation von HIF $\alpha$-Protein. Darüber hinaus konnten wir zeigen, dass HIF1 $\alpha$ transkriptionell durch NFאB (nuclear factor ,kappa-light-chain-enhancer" of activated B-cells) reguliert wird. Auch dieser Mechanismus wird durch NADPH-Oxidasen aktiviert [12].

Kürzlich konnten wir zeigen, dass die NADPH-Oxidase-HIF-Signalachse zur Ausbildung von kardiovaskulären Komplikationen, wie Hypertonie bei der Glukokortikoidvermittelten Stressantwort, beiträgt [14]. Dabei interagieren NADPH-Oxidasen und das HIF-System nicht nur unter normoxischen, sondern auch unter hypoxischen Bedingungen [15]. NADPH-Oxidasen sind daher auch an der Regulation von Hypoxie-induzierten zellulären Prozessen beteiligt, wie z. B. der Angiogenese.

Sie spielen aber auch eine Rolle bei der systemischen Hypoxieantwort [13]. Im Mausmodell führt eine reduzierte Sauerstoffkonzentration von zehn Prozent, die etwa der Sauerstoffkonzentration auf dem Kilimandscharo entspricht, nach einem Zeitraum von drei Wochen nicht nur zu pulmonal-vaskulärem Remodeling und zur Ausbildung eines Lungenhochdrucks, sondern auch zu einer Hochregulation von NADPH-Oxidasen und HIF $1 \alpha$.

Mäuse, denen eine funktionelle NADPHOxidase aufgrund einer Punktmutation im p22phox-Gen fehlt, zeigen keine Erhöhung von HIF1 $\alpha$ und sind vor der Entwicklung des Hypoxie-induzierten Lungenhochdrucks geschützt [13]. 


\section{Regulation der NADPH-Oxidasen durch Hypoxie und HIF}

Mechanistisch konnten wir zeigen, dass NADPH-Oxidase-Untereinheiten wie NOX4 und NOX2 HIF1-Zielgene [10, 16] sind und unter Hypoxie transkriptionell induziert werden. Damit ist HIF1 $\alpha$ auch direkt an der Regulation der NADPH-Oxidase abhängigen ROS-Bildung beteiligt.

Auch die Untereinheit p22phox wird unter hypoxischen Bedingungen heraufreguliert. Ähnlich wie HIF1 $\alpha$ wird p22phox unter normoxischen Bedingungen ubiquitiniert, wohl unter der Beteiligung von PHD2 und pVHL. Hypoxie vermindert die Ubiquitinierung und stabilisiert das p22phox-Protein [13].

Dieser Kopplungsmechanismus erlaubt eine Hochregulation von NADPH-Oxidasen und der Kapazität zur Produktion von ROS auch unter Hypoxie.

\section{Rolle der NADPH-Oxidasen unter Hypoxie - der physiologische „ROS-Korridor"}

Welche Rolle spielen NADPH-Oxidasen nun unter Hypoxie auf zellulärer Ebene? Wir denken, dass sie wichtig für die Aufrechterhaltung eines gewissen ROS-Tonus auch unter Hypoxie sind.

Das Ausmaß der ROS-Produktion unter Hypoxie wird noch immer kontrovers diskutiert [7]. Zum einen wird eine erhöhte ROSProduktion unter Hypoxie, die möglicherweise durch eine dysfunktionale Atmungskettenaktiviät in den Mitochondrien verursacht ist, berichtet. Zum anderen konnten wir und andere zeigen, dass ROS unter Hypoxie zunächst vermindert produziert werden, sofern die Messung unter Hypoxie stattfindet. Sobald jedoch nach Hypoxie oder während der Messung normoxische Verhältnisse eintreten, kommt es zu einer Reoxygenierung und dadurch zu vermehrter ROSBildung [17]. Inwieweit Befunde von erhöhten ROS-Spiegeln nach Hypoxie auf solche Reoxygenierungseffekte auch in anderen Studien zurückgeführt werden können, ist noch nicht endgültig geklärt.

Fehlen funktionelle NADPH-Oxidasen, so kommt es nicht nur zu einem Verlust der ROS-Bildung nach Reoxygenierung, auch die ROS-Bildung unter Hypoxie wird weiter vermindert. In Zellen, in denen NADPHOxidasen vermehrt exprimiert werden, ist der ROS-Abfall unter Hypoxie jedoch aufgehoben. Wir vermuten daher, dass die hypoxische Induktion von NADPH-Oxidasen u. a. dazu dient, als Gegenmaßnahme eine gewis- se ROS-Produktion auch unter hypoxischen Bedingungen zu gewährleisten. Wir gehen davon aus, dass es einen physiologischen „ROS-Korridor" gibt, innerhalb dessen viele zelluläre physiologische Prozesse funktionieren. Sowohl eine starke Verminderung von ROS - wie unter Hypoxie -als auch eine starke Erhöhung von ROS - wie bei Reoxygenierung oder anderen Prozessen - führt zu pathophysiologischen Reaktionen bis hin zu Zelltod durch Apoptose oder Nekrose. Durch zelluläre Gegenmaßnahmen - wie z. B. die Regulation der NOX- und HIF-Proteine - wird versucht, den ROS-Korridor wieder zu erreichen (Abb. 2B). Das komplexe Wechselspiel zwischen ROS-generierenden NADPH-Oxidasen und dem HIF-System scheint dabei eine wichtige Rolle in der Regulation der Redoxhomöostase innerhalb dieses ROS-Korridors zu spielen und bietet wichtige Zielstrukturen zur Behandlung von Erkrankungen mit dysregulierter ROS-Produktion und Verlust der Redoxhomöostase, wie Herzinfarkt, Diabetes oder Krebs.

\section{Literatur}

[1] Sies H, Jones DP (2020) Reactive oxygen species (ROS) as pleiotropic physiological signalling agents. Nat Rev Mol Cell Biol 21: 363-383

[2] Bedard K, Krause KH (2007) The NOX family of ROSgenerating NADPH oxidases: physiology and pathophysiology. Physiol Rev 87: 245-313

[3] Belambri SA, Rolas L, Raad H et al. (2018) NADPH oxidase activation in neutrophils: role of the phosphorylation of its subunits. Eur J Clin Invest 48 (Suppl 2): e12951

[4] Petry A, Gorlach A (2019) Regulation of NADPH Oxidases by G protein-coupled receptors. Antioxid Redox Signal 30: 74-94

[5] BelAiba RS, Djordjevic T, Petry A et al. (2007) NOX5 variants are functionally active in endothelial cells. Free Radic Biol Med 42: 446-459

[6] Petry A, Djordjevic T, Weitnauer M et al. (2006) NOX2 and NOX4 mediate proliferative response in endothelial cells. Antioxid Redox Signal 8: 1473-1484

[7] Görlach A, Dimova EY, Petry A et al. (2015) Reactive oxygen species, nutrition, hypoxia and diseases: problems solved? Redox Biol 6: 372-385
[8] Kietzmann T, Petry A, Shvetsova A et al. (2017) The epigenetic landscape related to reactive oxygen species formation in the cardiovascular system. Br J Pharmacol 174: 15331554

[9] Semenza GL (2014) Oxygen sensing hypoxia-inducible factors, and disease pathophysiology. Annu Rev Pathol 9: 47-71

[10] Diebold I, Petry A Sabrane K et al. (2012) The HIF1 target gene NOX2 promotes angiogenesis through urotensin-II. J Cell Sci 125: 956-964

[11] Diebold I, Flügel D, Becht S et al. (2010) The hypoxia-inducible factor-2alpha is stabilized by oxidative stress involving NOX4. Antioxid Redox Signal 13: 425-436

[12] Bonello S, Zähringer C, BelAiba RS et al. (2007) Reactive oxygen species activate the HIF-1alpha promoter via a functional NFkappaB site. Arterioscler Thromb Vasc Biol 27: 755761

[13] Zhang Z, Trautz B, Kracun D et al. (2019) Stabilization of p22phox by hypoxia promotes pulmonary hypertension. Antioxid Redox Signal 30: 56-73

[14] Kracun D, Klop M, Knirsch A et al. (2020) NADPH oxidases and HIF1 promote cardiac dysfunction and pulmonary hypertension in response to glucocorticoid excess. Redox Biol 34: 101536

[15] Belaiba RS, Bonello S, Zähringer C et al. (2007) Hypoxia up-regulates hypoxia-inducible factor-1alpha transcription by involving phosphatidylinositol 3-kinase and nuclear factor kappaB in pulmonary artery smooth muscle cells. Mol Biol Cell 18: 4691-4697

[16] Diebold I, Petry A, Hess J, Gorlach A (2010) The NADPH oxidase subunit NOX4 is a new target gene of the hypoxia-inducible factor-1. Mol Biol Cell 21: 2087-2096

[17] Chalupsky K, Kracun D, Kanchev I et al. (2015) Folic acid promotes recycling of tetrahydrobiopterin and protects against hypoxia-induced pulmonary hypertension by recoupling endothelial nitric oxide synthase. Antioxid Redox Signal 23: 1076-1091

Funding note: Open Access funding enabled and organized by Projekt DEAL. Open Access: Dieser Artikel wird unter der Creative Commons Namensnennung 4.0 International Lizenz veröffentlicht, welche die Nutzung, Vervielfältigung, Bearbeitung, Verbreitung und Wiedergabe in jeglichem Medium und Format erlaubt, sofern Sie den/die ursprünglichen Autor(en) und die Quelle ordnungsgemäß nennen, einen Link zur Creative Commons Lizenz beifügen un angeben, ob Änderungen vorgenommen wurden. Die in diesem Artikel enthaltenen Bilder und sonstiges Drittmaterial unterliegen ebenfalls der nichts anderes ergibt. Sofern das betreffende Material nicht unter der genannten Creative Commons Lizenz steht und die betreffende Handlung nicht nach gesetzlichen Vorschriften erlaubt ist, ist für die oben aufgeführten nach gesetzlichen Vorschriften erlaubt ist, ist für die oben aufgeführt
Weiterverwendungen des Materials die Einwilligung des jeweiligen Rechteinhabers einzuholen. Weitere Details zur Lizenz entnehmen Sie bitte der Lizenzinformation auf http://creativecommons.org/licenses/by/4.0/deed.de.

\section{Korrespondenzadresse:}

Dr. Andreas Petry

Experimentelle und Molekulare Kinderkardiologie Deutsches Herzzentrum München

Lazarettstraße 36

D-80636 München

petry@dhm.mhn.de

Andreas Petry
$\begin{aligned} & 1998-2003 \text { Biologiestudium an der Universität Frankfurt a. M. 2003-2009 Doktor- } \\ & \text { arbeit in der Experimentellen und Molekularen Kinderkardiologie (ExKK) bei Prof. Dr. } \\ & \text { A. Görlach am Deutschen Herzzentrum München der TU München mit Promotion zum } \\ & \text { Dr. rer. nat. } 2009 \text { an der Fakultät für Chemie und Pharmazie der LMU München. Seit } \\ & 2013 \text { Projektleiter der ExKK im Deutschen Herzzentrum München. }\end{aligned}$
$\begin{aligned} & \text { Agnes Görlach } \\ & \text { 1984-1990 Studium der Humanmedizin in München und Bochum. 1992 Promotion } \\ & \text { am Max-Planck-Institut für Molekulare Physiologie, Dortmund; Otto-Hahn-Medaille der } \\ & \text { Max-Planck-Gesellschaft. 1993-1996 Postdoc Scripps Research Institute, La Jolla, } \\ & \text { CA, USA. 1996-1998 Institut für Physiologie, Universität Zürich, Schweiz. 2001 } \\ & \text { Habilitation Physiologie, Universität Frankfurt a. M. Seit 2001 Leiterin Abtl. Experi- } \\ & \text { mentelle und Molekulare Kinderkardiologie, Deutsches Herzzentrum München an der } \\ & \text { TU München. Seit 2009 Professorin für Experimentelle und Molekulare Kinderkardio- } \\ & \text { logie an der TU München. }\end{aligned}$

